\section{A SYSTEMATIC REVIEW OF MUSCULOSKELETAL INJURIES IN PROFESSIONAL GOLFERS}

${ }^{1}$ Patrick Robinson, 'lain Murray, ${ }^{1}$ Andrew Duckworth, ${ }^{2}$ Roger Hawkes, ${ }^{2}$ Danny Glover, ${ }^{2}$ Nigel Tilley, ${ }^{2}$ Rob Hillman, ${ }^{1,3}$ Chris Oliver, ${ }^{2}$ Andrew Murray. ${ }^{1}$ Department of Orthopaedics and Trauma, Edinburgh, UK; ${ }^{2}$ European Tour Performance Institute, Virginia Water, UK; ${ }^{3}$ Department of Sports and Exercise/Physical Activity for Health, Edinburgh, UK

\subsection{6/bjsports-2021-IOC.246}

Background The distribution of professional golfing injuries is poorly understood.

Objective The aim of the study was to perform a systematic review to describe the epidemiology of musculoskeletal injuries in professional golfers.

Design A systematic review using Preferred Reporting Items for Systematic Review and Meta-Analysis (PRISMA) guidelines. The databases used were Pubmed, SportDiscus and Embase. The inclusion criteria was published observational research articles relating to the incidence or prevalence of musculoskeletal injuries in professional golfers, which were written in the English language and not restricted by age or gender.

Setting Professional golf.

Main Outcome Measurements Data collected included age, sex, data collection methods, diagnosis, region of injury, side of injury, incidence/prevalence of injury, definition of injury, nature of injury, severity of injury, mechanism of injury, risk factors, length of golfing career, injury management and time to return to sport.

Results Of the 1863 studies identified on the initial search, five studies were found to satisfy the inclusion criteria for analysis. The mean age of the golfers in these studies was 34.8 ( \pm 3.6 ) years. The gender of patients in included studies compromised $72 \%$ males and $28 \%$ females. Four studies reported that lumbar spine injuries were the most common (range 22-34\%). Excluding injuries to the spine (lumbar, thoracic and cervical), the hand/wrist was the next most common region of injury (range 6-37\%). The quality of the studies was relatively poor with no study satisfying $>50 \%$ of the quality assessment tool questions and only one study giving a clear definition of how they defined injury.

Conclusions There is a paucity of well-designed epidemiological studies evaluating musculoskeletal injuries affecting professional golfers. Injuries to the spine are the most frequently affected region, followed by the hand/wrist. This study has identified targeted areas of future research that aims to improve the management of injuries among professional golfers.

\section{A RANDOMISED CONTROLLED TRIAL INVESTIGATING THE CROSS-EDUCATION OF STRENGTH AND POWER FOLLOWING AT-HOME UNILATERAL CALF EXERCISES}

\footnotetext{
1,2Niall Simmons, 1,2Sumona Mandal, 1,2 Liang Zhi Wong, 1,2Andreas Mirallais, ${ }^{1}$ Flaminia Ronca, ${ }^{1}$ Bhavesh Kumar. ${ }^{1}$ The Institute of Sport Exercise and Health, London, UK; ${ }^{2}$ University College London, London, UK
}

\subsection{6/bjsports-2021-IOC.247}

Background Cross-education (CE) describes contralateral muscle function gain following unilateral limb training. CE has the potential to reduce strength and power losses following unilateral limb immobilisation. Despite the potential for wider rehabilitative application, few CE studies have utilised home-based interventions.

Objective Can unilateral home-based strength training elicit bilateral strength and power increases in the calf?

Design A prospective, randomised controlled trial utilising single-blinding.

Setting The Institute of Sport Exercise \& Health, London

Participants Thirty-five healthy participants (21.0 \pm 1.5 years) with no resistance training 6 months prior to the study were recruited; thirty-four completed the study.

Intervention Participants were randomised to Intervention $(n=20)$ or Control $(n=15)$. Intervention completed 8 weeks of unilateral straight and bent-knee calf raises at-home, using resistance tubing. Load was self-progressed, sustaining a BORG CR10 score of seven.

Main Outcome Measurements Concentric and eccentric calf peak torque was measured using isokinetic dynamometry at $30 \%$. Power output was measured during a single-leg jump using ChronoJump ${ }^{\circledR}$ (Chronojump-Boscosystem). Measurements were taken PRE, MID and POST-intervention.

Results The trained leg increased in strength PRE-POST for concentric $(25.8 \pm 29.3 \%, \mathrm{p}<0.01)$ and eccentric $(19.5 \pm 17.8 \%$, $\mathrm{p}<0.001)$ contractions. The untrained leg also increased in strength PRE-POST for concentric $(20.5 \pm 25.6 \%, \mathrm{p}<0.05)$ and eccentric $(14.8 \pm 19.2 \%, \mathrm{p}<0.05)$ contractions.

Power increased PRE-POST in the trained leg $(19.3 \pm 4.1 \%$, $\mathrm{p}<0.01)$. The untrained leg displayed PRE-POST power increases $(18.8 \pm 3.4 \%, \mathrm{p}<0.01)$. Controls displayed no significant strength or power changes.

In the untrained leg, strength increases were significant PRE-MID $(16.3 \pm 16.9 \%, \mathrm{p}<0.01)$ but not MID-POST (1.3 $\pm 13.7 \%, \mathrm{p}>0.05)$. The reverse was true for power with significant increases MID-POST $(10.3 \pm 12.6 \%, \mathrm{p}<0.01)$ but not PRE-MID (8.3 $\pm 13.3 \%, \mathrm{p}>0.05)$.

Conclusions This study demonstrates that unilateral, homebased strength training elicits significant bilateral calf strength and power increases. Additionally, the CE of strength seen prior to power indicates that outcomes secondary to the training type require greater training durations to reach significance. These findings may have rehabilitative potential, however further work is required in clinical populations.

\section{COMMERCIALLY-AVAILABLE INERTIAL MEASUREMENT UNIT UNDERESTIMATES NUMBER OF JUMPS FOR FEMALES MORE THAN MALES: IMPLICATIONS FOR LOAD MONITORING AND INJURY PREVENTION}

${ }^{1}$ Tyler J Tait, ${ }^{1}$ Lauren C Benson, ${ }^{1}$ Kimberley Befus, ${ }^{1}$ John Choi, ${ }^{1}$ Colin Hillson, ${ }^{1}$ Carlyn Stilling, ${ }^{1}$ Sagar Grewal, ${ }^{2}$ Kerry MacDonald, ${ }^{1,3,4,5}$ Kati Pasanen, 1,4,5,6,7 Carolyn Emery. 'Sport Injury Prevention Research Centre, Faculty of Kinesiology, University of Calgary, Calgary, Canada; ${ }^{2}$ Volleyball Canada, Ottawa, Canada; ${ }^{3}$ Tampere Research Center of Sports Medicine, UKK Institute, Tampere, Finland; ${ }^{4}$ Alberta Children's Hospital Research Institute, University of Calgary, Calgary, Canada; ${ }^{5}$ McCaig Bone and Joint Institute, Cumming School of Medicine, University of Calgary, Calgary, Canada; ${ }^{6}$ Department of Community Health Sciences, Cumming School of Medicine, University of Calgary, Calgary, Canada; 'Department of Pediatrics, Cumming School of Medicine, University of Calgary, Calgary, Canada

\subsection{6/bjsports-2021-IOC.248}

Background A high incidence of overuse knee injuries among youth basketball players may be attributed to improperly- 